\title{
SOLUTION BY RELAXATION METHODS OF PLANE POTENTIAL PROBLEMS WITH MIXED BOUNDARY CONDITIONS*
}

\author{
BY \\ L. FOX \\ Imperial College of Science and Technology, London
}

1. Introduction. The method of relaxation, as originally propounded by Southwell [1], was used to calculate the stresses in braced frameworks. A physical picture of the method, as presented by him in the Wright Brothers Memorial Lecture for 1941 , is the following. At each joint of the structure constraints are applied which prevent joint displacements and bear all the load. One constraint is then relaxed, thereby transferring some of its load to the members of the framework and some to adjacent constraints. Each constraint is relaxed in turn, and more of the load is imposed on the framework, until the residual loads (still borne by the constraints) may be deemed negligible.

In a series of eight papers [2:I-VIII], Southwell and collaborators have applied relaxation methods to various engineering problems. In some of these the method is applied to two-dimensional problems [2:III], and solutions are obtained of the equation

$$
\nabla^{2} w=Z
$$

for any boundary on which $w$ is prescribed, $Z$ being a given function of $x$ and $y$. Here Prandtl's membrane analogy [3] is used, in which $w$ is the displacement of a membrane fastened at its boundary and acted upon by a transverse force $Z$. The membrane is replaced by a mesh of uniformly tensioned strings, the mesh lines forming squares or equilateral triangles, and the tension in the strings being proportional to the surface tension of the membrane. Initially the mesh is flat and the load $Z$ is taken by constraints acting at the mesh points. The constraints are relaxed one by one, just as in the framework, until the loads are all taken by the strings, and the resulting displacements of the mesh points are recorded. Evidently, as the meshlength decreases, the approximation of the mesh to the continuous membrane is improved.

In a recent paper [2:VIII], Southwell and Vaisey extend the membrane-net analogy to obtain solutions of Laplace's equation in the case when the normal gradient $\partial w / \partial \nu$, instead of the function $w$, is given at the boundary. Here $\partial w / \partial \nu$ is regarded as a line intensity of transverse loading applied round the boundary of the membrane. This load is then integrated and distributed statically to the strings which cross the boundary.

There is a mathematical treatment of the above problems, based on finite differences. For Laplace's problem of the first kind, in which the function is specified on the boundary, the finite difference equations have been derived [2:III]. They are in general identical with the equations obtained from the analogy of membranes and tensioned nets.

\footnotetext{
* Received May 19, 1944.
} 
The corresponding mathematical treatment of Laplace's problem of the second kind, however, in which the normal gradient of the function is given on the boundary, was not given by Vaisey and Southwell, and it is this treatment with which this paper is concerned. The finite-difference equations are substantially different from those obtained in their paper.

The technique presented here would seem to be particularly desirable for problems in which the boundary conditions involve both the value of the function and its normal gradient. For then the mechanical analogy becomes somewhat complicated, especially in the case of solids of revolution, for which the analogy of variably tensioned nets is not attractive.

2. The finite difference approach to the relaxation method. For square meshes (Fig. 1), we have the approximations

$$
\begin{gathered}
2 a \frac{\partial w}{\partial x} \doteqdot w_{1}-w_{3}, \quad a^{2} \frac{\partial^{2} w}{\partial x^{2}} \doteqdot w_{1}+w_{3}-2 w_{0}, \\
a^{2} \nabla^{2} w \doteqdot w_{1}+w_{2}+w_{3}+w_{4}-4 w_{0} .
\end{gathered}
$$

Similar formulae are easily obtainable for triangular meshes. The order of the error in these approximations is known in each case, and decreases with the mesh-length $a$.

There is a finite-difference equation of type (2) for every mesh point, and the solution of these equations gives a numerical value of $w$ at each mesh point.

At points close to a curved boundary, such as 0 in Fig. 2, we obtain the finitedifference equation as follows, in the case when $w$ is given on the boundary. The

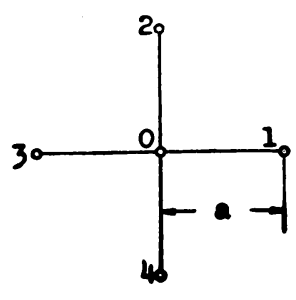

Fig. 1.

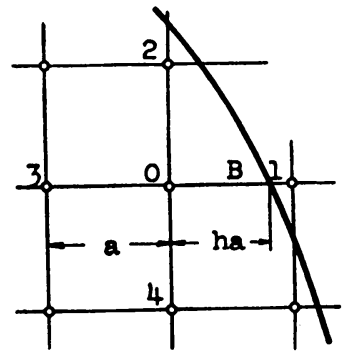

FIG. 2.

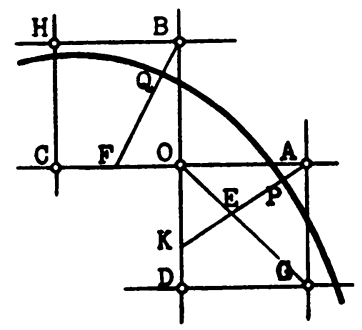

Fig 3.

arm 01 passes outside the boundary, and a linear interpolation along 01 yields $w_{B}=w_{D}+\left(w_{1}-w_{0}\right) h$, from which we obtain for the finite-difference equation at 0

$$
w_{2}+w_{3}+w_{4}+\frac{w_{B}}{h}-\left(3+\frac{1}{h}\right) w_{0}=0 .
$$

All these formulae are reproduced identically by the net analogy, but a more accurate formula than (3) given by Christopherson (4), and obtained by a parabolic interpolation, has not been deduced by analogy.

When $\partial w / \partial \nu$, rather than $w$, is given on the boundary, the same general equations hold as before, but there is a different procedure for points adjacent to the boundary. To write down the finite-difference equation at the point 0 (Fig. 3), we require $w_{A}$ and $w_{B}$. For $w_{A}$, we draw the normal APE and use the approximation 


$$
\mathrm{AE} \cdot\left(\frac{\partial w}{\partial \nu}\right)_{P}=w_{A}-w_{E} .
$$

A linear interplation along OG gives

$$
\mathrm{OG} \cdot w_{E}=\mathrm{OE} \cdot w_{G}+\mathrm{EG} \cdot w_{0},
$$

and elimination of $w_{B}$ between these two equations yields

$$
w_{A}=\frac{\mathrm{OE}}{\mathrm{OG}} w_{s}+\frac{\mathrm{OE}}{\mathrm{OG}} w_{O}+\mathrm{AE}\left(\frac{\partial w}{\partial \nu}\right)_{P} .
$$

Similarly, we obtain $w_{B}$ by drawing the normal $\mathrm{BQF}$.

The normal can be terminated on any convenient line. Thus in Fig. 3 we could produce $\mathrm{AE}$ to $\mathrm{K}$ on $\mathrm{OD}$, to obtain $w_{A}$ in terms of $w_{O}, w_{D}$, instead of $w_{O}, w_{G}$. The shorter the normal, however, the more accurate is the approximation (4), so in this case $\mathrm{E}$ is the best place to stop the normal. On the other hand, the normal from $B$ is continued to $F$, because a termination on the diagonal $\mathrm{HO}$ would involve the value $w_{H}$, itself fictitious, and the calculation would become more cumbersome.

The approximations employed to date have assumed $w$ to be linear along any line near the boundary. Improvements in accuracy can be made at the cost of additional labour. Thus, if the normal is stopped so that it is bisected by the boundary (Fig. 4), the formula

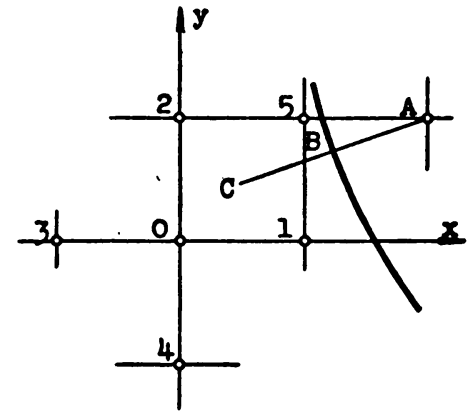

Fig. 4.

$$
\mathrm{AC} \cdot\left(\frac{\partial w}{\partial \nu}\right)_{B}=w_{A}-w_{C}
$$

assumes a parabolic variation of $w$. The point $C$ in general no longer lies on a diagonal or mesh line, but its value can easily be found, by double interpolation, to the approximation of Eq. (5). This procedure yields a higher accuracy, but it is better, except when a very high accuracy is required, to use a linear variation together with a finer mesh.

3. Problem I. Let us find the function $w$, harmonic in the circle

$$
x^{2}+y^{2}-2 x-2 y+1=0,
$$

and satisfying the boundary condition

$$
\frac{\partial w}{\partial \nu}=\frac{y-x}{x^{2}+y^{2}} .
$$

This is one of the problems attacked by Vaisey and Southwell [2:VIII]. It has the exact solution

$$
w=\tan ^{-1} y / x
$$


whence we have a guide to the accuracy of our method. The solution is unique, except for an arbitrary constant, and we choose the constant so that $w=\pi / 4$ at the centre of the circle.

First, we take the mesh-length equal to the radius of the circle. The mesh contains only five points. Fig. 5 shows this mesh, the finite-difference equations, and the values of $w$ multiplied by 1000 . External mesh points are denoted by open circles. For comparison, the exact values are entered under the approximate values.

When the mesh-length is halved, there are twelve fictitious mesh points and thirteen simultaneous equations. The solution is given in Fig. 6.

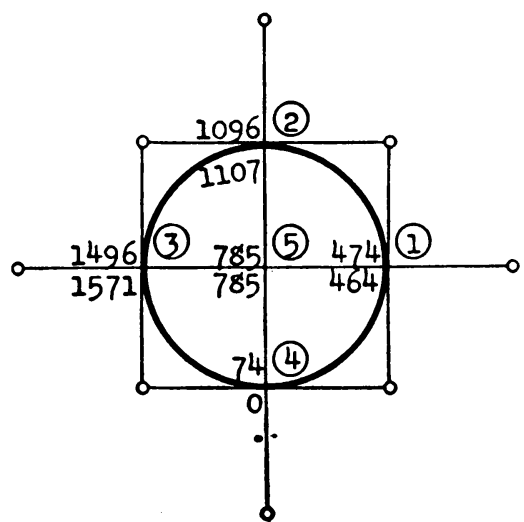

Fig. 5.
$2(5)+.5(2)+.5(4)-3(1)-733=0$

$2(5)+.5(1)+.5(3)-3(2)+733=0$

$2(5)+.5(2)+.5(4)-3(3)+2333=0$

$2(5)+.5(1)+.5(4)-3(4)-2333=0$

$(1)+(2)+(3)+(4)-4(5)=0$

As the form of $\partial w / \partial \nu$ indicates, $w$ is skew-symmetrical about the line $x=y$. This feature was not utilized in construction of Figs. 5 and 6 , but it is found useful as a labour-saving device in the case of a mesh-length of one-quarter of the radius. The

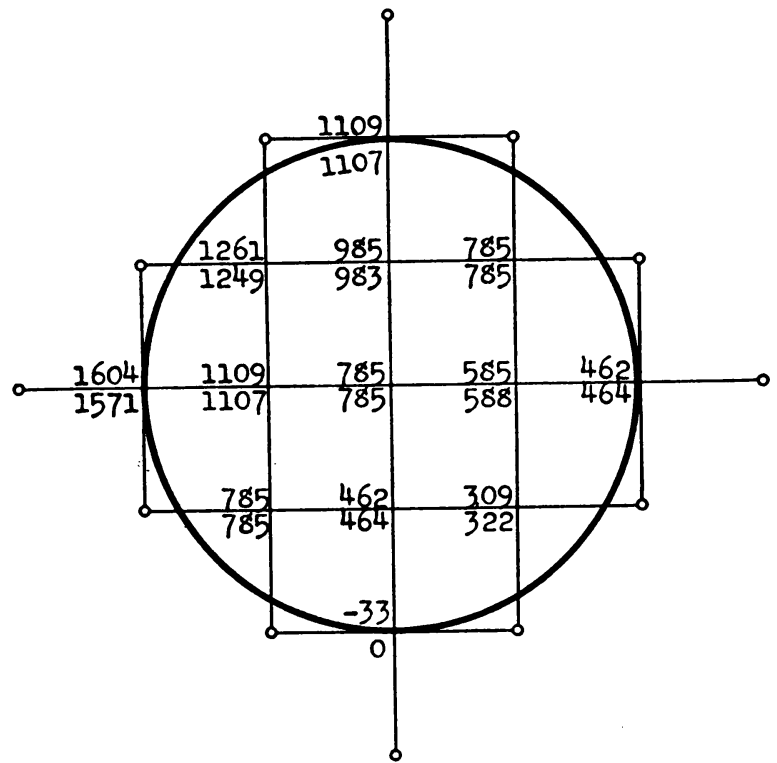

Fig. 6. 
results in this case are shown in Fig. 7, and are very close to those for the exact solution, the error being greatest at points nearest the origin. This was to be expected, since $\partial w / \partial \nu$ changes rapidly in value across the line $x=y$ when $x^{2}+y^{2}$ is small. Comparable errors were found in the treatment by Vaisey and Southwell.

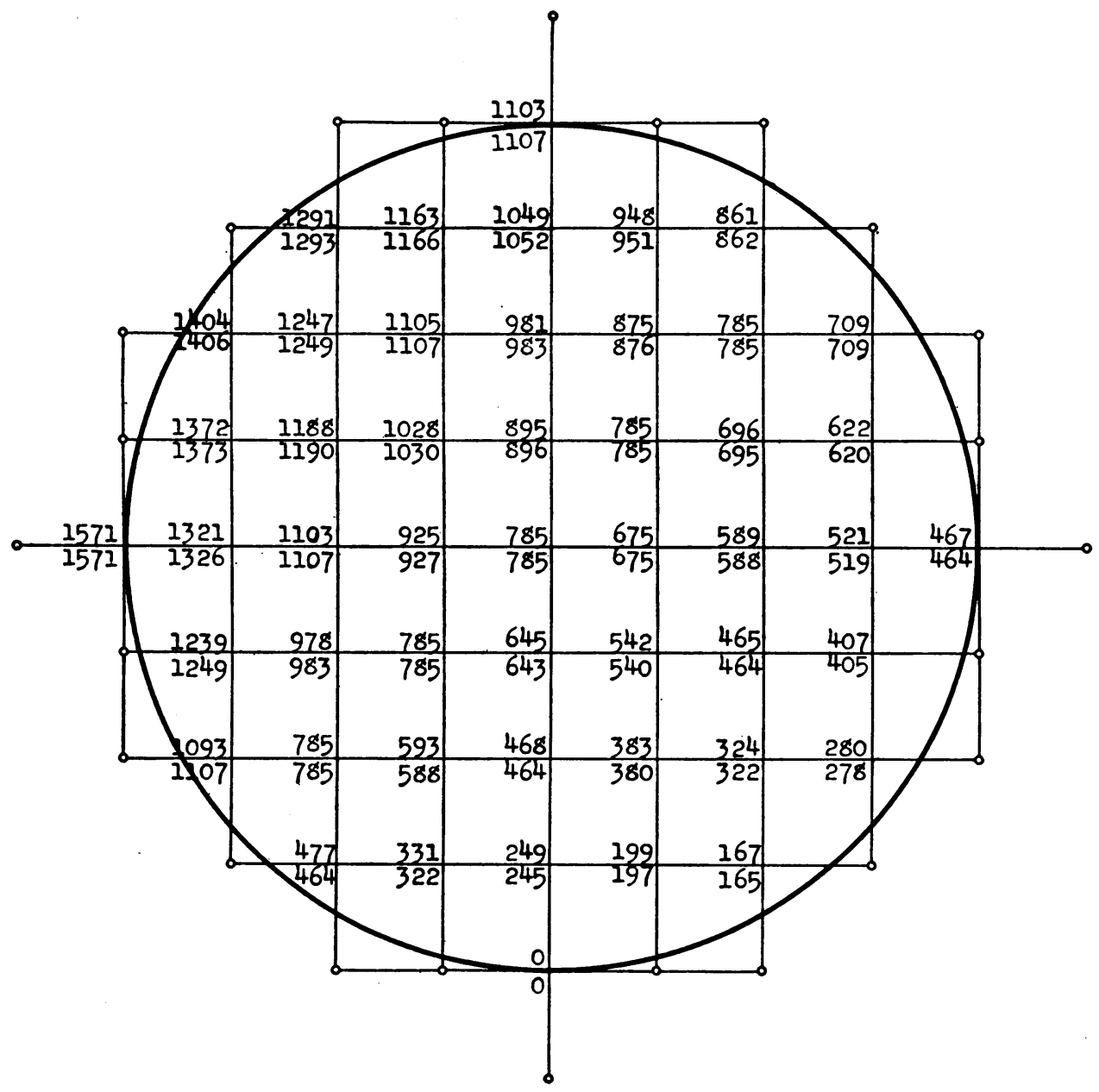

Fig. 7.

An interesting point is the oscillatory nature of the convergence of values of $w$. In the boundary-value-specified problem, values usually converge from one side only.

4. Problem II. Let us find the function $w$, harmonic in the same circle as before, but satisfying the boundary condition

$$
\left(\frac{\partial}{\partial \nu}-\frac{1}{r}\right) w=\frac{1}{r^{2}}\left(y-x-r \tan ^{-1} \frac{y}{x}\right),
$$

where $r=\sqrt{x^{2}+y^{2}}$. The exact solution is again $w=\tan ^{-1} y / x$.

Here the boundary condition involves the value as well as the normal slope of 
the function, but only a slight extension of the method of the previous problem is needed. As before, fictitious points are eliminated from the governing equation by means of the boundary condition.

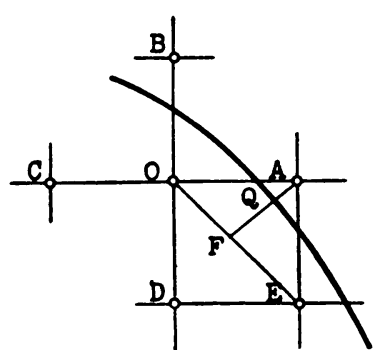

Fig. 8.

In order to write down the finite-difference equation at 0 (Fig. 8), we need $w_{A}$ and $w_{B}$. As before, the normal AF yields

$$
\mathrm{AF} \cdot\left(\frac{\partial w}{\partial \nu}\right)_{Q}=w_{A}-w_{F},
$$

and linear interpolation on $\mathrm{OE}$ and $\mathrm{AF}$ gives

$$
\begin{aligned}
& \mathrm{OF} \cdot w_{F}=\mathrm{OF} \cdot w_{E}+\mathrm{FE} \cdot w_{O}, \\
& \mathrm{AF} \cdot w_{Q}=\mathrm{AP} \cdot w_{F}+\mathrm{PF} \cdot w_{A} .
\end{aligned}
$$

From these three equations and the boundary condition (7), $w_{A}$ can be found in

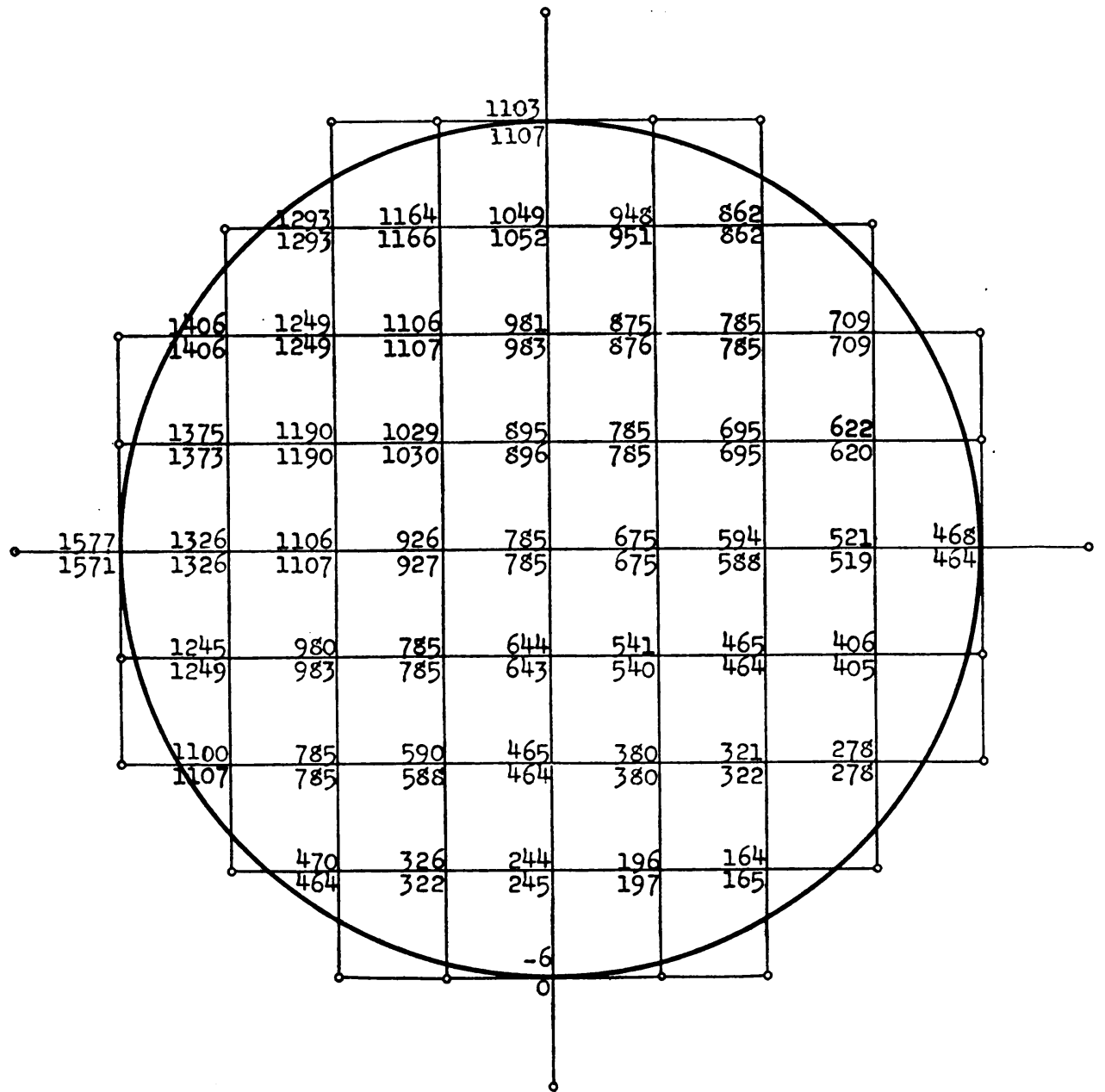

Fig. 9. 
terms of $w_{O}$ and $w_{E}$. A similar operation yields $w_{B}$, and hence the finite-difference equation for the point $\mathrm{O}$ can be written down, and the problem solved.

As in the previous problem it was found that decrease of the mesh-length results in oscillatory convergence. The oscillation is rather more violent, but the final result shown in Fig. 9 is no less accurate, notwithstanding the additional interpolation.

5. Summary. In this paper solutions are obtained of Laplace's equation with boundary condition involving either the normal gradient only, or both the boundary value and the normal gradient. The need for a technique for problems of this kind has arisen in recent developments of the relaxation method. Two problems are solved, both have a known analytical solution, and good results are obtained in each case. The method used is independent of the analogy of tensioned nets, and can be applied without modification to problems for which analogies may be difficult to use.

\section{REFERENCES}

[1] R. V. Southwell, Proc. Roy. Soc. Lon. (A), 151, 59-65 (1935); 153, 41-76 (1935).

[2] Relaxation methods applied to engineer.ng problems:

I. K. N. E. BRADFIELd and R. V. Southwell, Deflection of beams under transverse loading, Proc. Roy. Soc. Lon. (A), 161, 155-181 (1937).

II. A. N. BlACK and R. V. Southwell, Basic theory, with applications to surveying and to electrical networks, and an extension to gyrostatic systems, Proc. Roy. Soc. Lon. (A), 164, 447-467 (1938).

III. D. G. Christopherson and R. V. Southwell, Problems involving two independent variables, Proc. Roy. Soc. Lon. (A), 168, 317-350 (1938).

IV. K. N. E. Bradfield, D. G. Christopherson and R. V. Southwell, Elastic stability and vibrations, Proc. Roy. Soc. Lon. (A), 169, 289-317 (1939).

V. R. W. G. GANDy and R. V. Southwell, Conformal transformation of a region in plane space, Trans. Roy. Soc. Lon. (A), 238, 453-475 (1940).

VI. A. Pellew and R. V. Southwell, Natural frequencies of systems having restricted freedom, Proc. Roy. Soc. Lon. (A), 175, 262-290 (1940).

VII. F. S. Shaw and R. V. Southwell, Problems relating to the percolation of fluids through porous materials, Proc. Roy. Soc. Lon. (A), 178, 1-17 (1941).

VIII. G. VAISEy and R. V. Southwell, Plane-potential problems involving specified normal gradients, Proc. Roy. Soc. Lon. (A), 182, 129-151 (1943).

[3] L. Prandtl, Physik. Z., 4, 758-759 (1903).

[4] D. G. Christopherson, Trans. A.S.M.E., 62, A1-A4 (1940). 